Proc. Indian Acad. Sci. (Chem. Sci.), Vol. 102, No. 5, October 1990, pp. 601-605

(C) Printed in India.

\title{
Einstein-like phonons in InSe
}

\author{
K IMAI*, K KUMAZAKI, S SETO ${ }^{1}$, K YAMAYA ${ }^{2}$ and Y ABE ${ }^{2}$ \\ Hokkaido Institute of Technology, Teine, Sapporo 006, Japan \\ ${ }^{1}$ Electronics Materials Laboratory, Sumitomo Metal Mining Co. Ltd., Suehiro, Ohme 198, \\ Japan \\ ${ }^{2}$ Department of Nuclear Engineering, Hokkaido University, Sapporo 060, Japan
}

\begin{abstract}
The specific heat of InSe obeys the Debye model in the temperature region between 2 and $4.5 \mathrm{~K}$. But it is explained as a superposition of the Debye and the Einstein models above $4.5 \mathrm{~K}$. The Debye and the Einstein temperatures are 185 and $58 \mathrm{~K}$, respectively. This Einstein temperature equals $40 \mathrm{~cm}^{-1}$ and corresponds to the energy of $E^{2}$ mode observed in the Raman scattering experiments.
\end{abstract}

Keywords. Debye temperature; Einstein temperature; InSe specific heat; Raman scattering experiments; Einstein-like phonons.

\section{Introduction}

InSe is one of the layer-type III-VI semiconductor compounds which have very characteristic structures. The bond between the fundamental layers is weak because of a van der Waals type force. The fundamental layer consists of four-fold sheets by a covalent bond such as Se/In/In/Se. The In-In bond has an anisotropy (Kuroda et al 1981; Riede et al 1982) such that the component of the force constant parallel to the $c$ axis is comparable to the $\mathrm{Se}$-In bond, and that of the perpendicular one is smaller by one order than that of the component parallel to the $c$ axis. It is expected that these characteristic structures influence the mechanical properties and the lattice vibrations.

An anisotropy of the mechanical properties appears in the elastic constants. $C_{66} / \mathrm{C}_{44}$ is a tentative criterion for evaluating the anisotropy because this corresponds to the squared ratio of the longitudinal to the transverse sound velocity along the $c$ axis. For InSe, since the $c$ direction is not the pure mode axis of the transverse elastic wave, we cannot easily use this ratio for estimating the amount of anisotropy. Instead, we can estimate the ratio of $\mathrm{C}_{11} / \mathrm{C}_{33}$ and its value of InSe is 2.0 ( $\mathrm{K}$ Imai and $\mathrm{Y}$ Abe, unpublished data; $C_{11}=7.5, C_{12}=-5.8, C_{14}=0.37, C_{33}=3.8$ and $C_{44}=2.5 \times 10^{10} \mathrm{~N} / \mathrm{m}^{2}$. These were obtained by the ultrasonic pulse-echo method at $77 \mathrm{~K}$ ), lower than that of $\mathrm{GaSe}(2.63$ or 2.94) (Gatulle et al 1983) and GaS (3.20 or 4.39) (Gatulle et al 1983) with similar structures. This means that the anisotropy of InSe is smaller than that of other III-VI layer semiconductors.

InSe does not have a rigid layer mode but a half layer shear mode (Polian et al 1976), because the crystal symmetry of InSe grown by the non-stoichiometric

\footnotetext{
* For correspondence
} 
Bridgman method (Imai et al 1981) is the rhombohedral $\left(C_{3 v}^{5} \gamma\right.$-phase) type. These are characteristic modes in layer type crystals. In this structure, a unit cell contains three layers. We can also consider, however, that the crystal has triclinic symmetry, the unit cell of which is in one layer. The unit cell includes two InSe molecules.

If the dispersion of the half layer shear mode is small, we should observe the Einstein-like lattice vibration with low energy. In this work, we report the temperature dependence of the specific heat of InSe for the first time. Consequently our aim was to recognize the existence of low energy phonons which obey the Einstein model from the data of the specific heat. We measured the Raman scattering spectra of the InSe single crystals to determine the frequency of the phonons which contribute to the specific heat. We confirm that the Einstein-like phonon is related to the Raman scattering peak.

\section{Experiments}

The InSe crystals were grown by the non-stoichiometric Bridgman method. The composition of the starting liquid phase was $\operatorname{In}_{1.04} \mathrm{Se}_{0.96}$ melt (Imai et al 1981). The purity of the substances was $6 \mathrm{~N}$. The crystal grown by this method was of the $n$-type.

Specific heat measurements were carried out by the thermal relaxation method (Early et al 1981) using an SOS (silicon on sapphire) bolometer. The sample was stuck to the bolometer using Thermal Compound (Wakefield). The typical size of samples for measuring the specific heat was about $5 \times 3 \times 0.5 \mathrm{~mm}^{3}$ and the weight was about $50 \mathrm{mg}$. The weights of the SOS bolometer and the Thermal Compound used in this work were 46.3 and $2.0 \mathrm{mg}$, respectively. The bolometer was set in a vacuum adiabatic cell. The thermal relaxation curve was measured by the a.c. modulation method. The lower limit of the measurable thermal capacity was $3.2 \times 10^{-6} \mathrm{~J} / \mathrm{K}$ at $4.2 \mathrm{~K}$ in this system. The temperature range was between 2 and $9 \mathrm{~K}$.

The Raman scattering measurements were performed at room temperature under $x\left(y,{ }_{z}^{y}\right) x$ using the Ramaner U-1000. The light source was an $\mathrm{Ar}^{+}$laser (488 nm) with an output power of $100 \mathrm{~mW}$. Sample treatment for the Raman scattering measurements was as follows: We cut out samples from the ingots to form a rectangular piece with a typical $c$ plane area of $7 \times 5 \mathrm{~mm}^{2}$. We pasted the sample to a $1 \mathrm{~cm}^{2} \mathrm{Cu}$ plate with an $\mathrm{In}-\mathrm{Hg}$ alloy and annealed this at $150^{\circ} \mathrm{C}$ under $10^{-3}$ Torr for $2 \mathrm{~h}$ to evaporate $\mathrm{Hg}$. We carefully cleaved the $c$ plane of the stuck crystal till the mirror-like surface appeared.

\section{Results and discussion}

Figure 1 shows $C / T$ as a function of $T^{2}$, where $C$ is the measured specific heat. Since InSe is a semiconductor, the specific heat is 0 at $0 \mathrm{~K}$. $C / T$ increases with increasing temperature. The temperature dependence of $C$ below $4.5 \mathrm{~K}$ agrees well with the Debye model. Above $4.5 \mathrm{~K}$, however, $C / T$ deviates remarkably from the Debye's $T^{3}$ law. This deviation enlarges with increasing temperature. We name the Debye part $C_{D}$ and the amount of deviation $C_{E}$ as shown in figure 1.

$C_{E}$ is plotted in figure 2 as $\operatorname{In}\left(C_{E} T^{2}\right)$ vs $1 / T$. The $C_{E}$ points lie in a straight line. This means that $C_{E}$ agrees with the Einstein model. It is considered that $C$ is the 


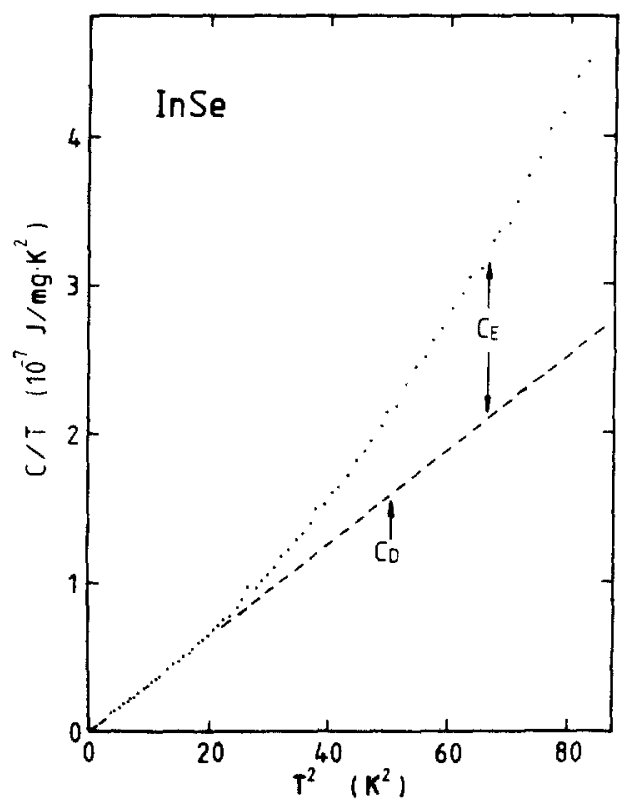

Figure 1. Temperature-dependence of specific heat. The dashed line is the specific heat which obeys the Debye model with a Debye temperature of $185 \mathrm{~K}$.

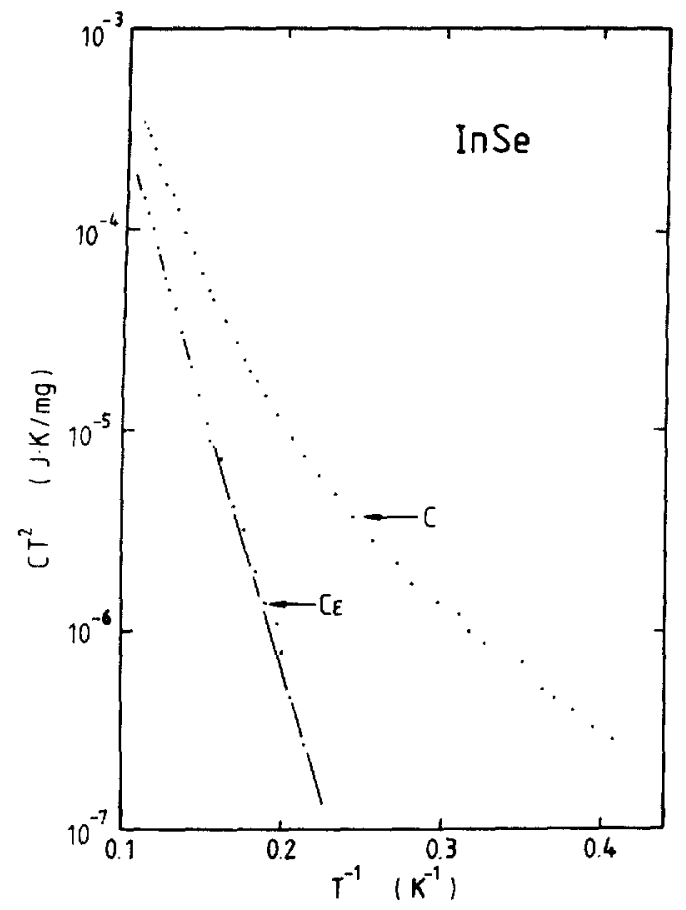

Figure 2. Logarithmic specific heat as a function of $1 / T$. The dashed line is the temperaturedependence as the Einstein model with an Einstein temperature of $58 \mathrm{~K}$. 


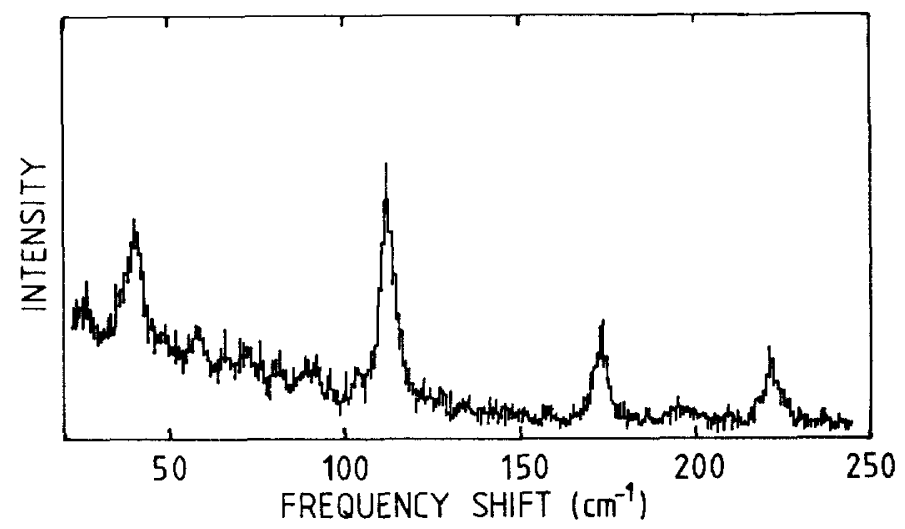

Figure 3. Typical Raman scattering spectrum of $y$-InSe at room temperature.

superposition of $C_{D}$ due to the Debye model and $C_{E}$ due to the Einstein model.

The Debye and Einstein temperatures estimated from figures 1 and 2 equal 185 and $58 \mathrm{~K}$, respectively. The Debye temperature is very close to the reported value of $190 \mathrm{~K}$ (Iskender-Zade et al 1977), which was calculated from the results of sound velocity measurements of $\beta^{-}$InSe (with $D_{6 h}^{3}$ symmetry).

Figure 3 is a typical Raman scattering spectrum. In comparison with the Raman scattering and IR absorption studies (Carlone et al 1981; Kuroda et al 1981; Riede et al 1982), we can assign the peaks at 40 and $117 \mathrm{~cm}^{-1}$ to the transverse optics (TO) and longitudinal optics (LO) modes, respectively. The TO mode is the half layer shear mode in $\gamma$-InSe as mentioned above. The Einstein temperature, $58 \mathrm{~K}$, corresponds to $40 \mathrm{~cm}^{-1}$. This energy agrees with the TO mode. It is confirmed that characteristic phonons in the layer type material contribute strongly to the specific heat.

There is a probability that the deviation from the Debye $T^{3}$ law is due ot the Schottky anomaly because InSe is a semiconductor. The temperature-dependence of the specific heat due to the Schottky anomaly and of the Einstein model are similar at low temperatures. If the deviation is due to the Schottky anomaly, the activation energy is about $5 \mathrm{meV}$. This value is very small because the donor state in InSe lies at about $18 \mathrm{meV}$ (Cingolani et al 1986) from the band edge. Therefore we conclude that the deviation of the activation energy $\approx 5 \mathrm{meV}$ in figure 1 is due to the Einstein-like phonons.

In summary, the thermal property of InSe obeys the Debye model in temperature regions lower than $4.5 \mathrm{~K}$. The Debye temperature of InSe is $185 \mathrm{~K}$. Between 4.5 and $9 \mathrm{~K}$, the half layer rigid mode with an energy of $40 \mathrm{~cm}^{-1}$ plays a great role in the thermal property based on the Einstein model. The anisotropy of InSe in the elastic and the electrical properties is weak. The temperature dependence of the electron Hall mobility in InSe indicates $T^{-1 \cdot 5}$ in the high temperature region (Segura et al 1984). It is due to the scattering with three-dimensional acoustic phonons and not to two-dimensional acoustic, polar optical, or homopolar optical modes (Fivaz and Mooser 1967). But it is clear that the characteristics of the layer material influence the macroscopic thermal property. 


\section{Acknowledgements}

The present work was supported in part by scientific research grants from the Private School Promotion Foundation, the Suhara Memorial Foundation and the Hokkaido Institute of Technology Foundation.

\section{References}

Carlone C, Jandl S and Shanks H R 1981 Phys. Status Solidi B103 123

Cingolani A, Cingolani R, Ferrara M and Lugara M 1986 Solid State Commun. 5763

Early S R, Hellman F, Marshall J and Geball T H 1981 Physica B\&C107 327

Fivaz R and Mooser E 1967 Phys. Rev. 163743

Gatulle M, Fischer M and Chevy A 1983 Phys. Status Solidi B119 327

Imai K, Suzuki K, Haga T, Hasegawa Y and Abe Y 1981 J. Cryst. Growth 54501

Iskender-Zade Z A, Faradzhew V D and Agaev A I 1977 Sov. Phys. Solid State 19492

Kuroda N, Nishina Y, Iwasaki H and Watanabe Y 1981 Solid State Commun. 38139

Polian A, Kunc K and Kuhn A 1976 Solid State Commun. 191079

Riede V, Neumann H, Levy F and Sobotta H 1982 Phys. Status Solidi B109 275

Segura A, Pomer F, Cantarero A, Krause W and Chevy A 1984 Phys. Rev. B29 5708 